\title{
Fechamento de capital e sua relação com as práticas de gerenciamento de resultados
}

\author{
Delisting and its relation to earnings management practices
}

La exclusión de las empresas del mercado de capitales y su relación con las prácticas de gestión de resultados

Recebido: 23/03/2021 | Revisado: 03/04/2021 | Aceito: 05/04/2021 | Publicado: 15/04/2021

Edson Carlos Cordeiro Júnior

ORCID: https://orcid.org/0000-0001-9908-909X Instituto Federal de Educação, Ciência e Tecnologia do Triângulo Mineiro, Brasil E-mail: edsoncarloscjr@gmail.com

Fernanda Cristina Costa Lopes

ORCID: https://orcid.org/0000-0001-7013-5793 Instituto Federal de Educação, Ciência e Tecnologia do Triângulo Mineiro, Brasil E-mail: fernandalopes@iftm.edu.br

Pablo Rogers

ORCID: https://orcid.org/0000-0002-0093-3834

Universidade Federal de Uberlândia, Brasil E-mail: pablorogers@ufu.br

\begin{abstract}
Resumo
Este estudo pretende explicar duas temáticas importantes e conexas para o mercado de capitais nacional: gerenciamento de resultado e deslistagem. Isto se deve a uma tentativa de encontrar padrões que expliquem a importância da qualidade da informação contábil e que auxiliem os gestores e stakeholders quanto às possíveis tomadas de decisões sobre o mercado. Este artigo pretende alcançar seu objetivo ao relacionar gerenciamento de resultados e deslistagem. Para isso, primeiramente foi feita uma regressão baseada no modelo de Kang e Sivaramakrishnan (1995), para se mensurar os accruals discricionários como proxy de gerenciamento de resultados. Em termos significativos, este artigo encontrou relação das variáveis de controle tamanho e endividamento com o gerenciamento de resultados e, consequentemente, com a qualidade da informação contábil, alinhando-se a literatura existente. Porém, não foi possível identificar na amostra estudada significância entre gerenciamento de resultados e deslistagem.
\end{abstract}

Palavras-chave: Fechamento de capital; Gerenciamento de resultados; Qualidade da informação contábil.

\begin{abstract}
This study intends to explain two important and related topics for the national capital market: earnings management and delisting. This is due to an attempt to find standards that explain the importance of the quality of accounting information and to assist managers and stakeholders in making market decisions. This article aims to achieve its goal when relating results management and delisting. For this, a regression was first made based on the model of Kang e Sivaramakrishnan (1995), to measure discretionary accruals as a proxy for results management. In significant terms, this article found a relationship between control variables size and indebtedness with the management of results and, consequently, with the quality of accounting information, aligning with the existing literature. However, it was not possible to identify in the studied sample the significance between results management and delisting.
\end{abstract}

Keywords: Delisting; Earnings management; Earnings quality.

\section{Resumen}

Este estudio pretende explicar dos temas importantes y relacionados para el mercado de capitales nacional: la gestión de resultados y la exclusión de empresas de la bolsa de valores. Esto se debe a un intento de encontrar estándares que expliquen la importancia de la calidad de la información contable y para ayudar a los gerentes y partes interesadas a tomar decisiones de mercado. Este artículo tiene como objetivo lograr su objetivo al relacionar la gestión de resultados y la exclusión de empresas de la bolsa de valores. Para ello, primero se realizó una regresión basada en el modelo de Kang e Sivaramakrishnan (1995), para medir las acumulaciones discrecionales como proxy de la gestión de resultados. En términos significativos, este artículo encontró una relación entre el tamaño de las variables de control y el endeudamiento con la gestión de resultados y, en consecuencia, con la calidad de la información contable, alineándose con la literatura existente. Sin embargo, no fue posible identificar en la muestra estudiada la significancia entre la gestión de resultados y la exclusión de empresas de la bolsa de valores.

Palabras clave: Exclusión de empresas de la bolsa de valores; Gestión de resultados; Calidad de resultados. 


\section{Introdução}

Qual o motivo para entrada no mercado de capitais? Segundo Pour e Lasfer (2013) as empresas entram no mercado para reequilibrar sua alavancagem e decidem sair quando não conseguem aproveitar as oportunidades de crescimento e aumento da rentabilidade. Para Pagano, Panetta e Zingales (1998) e Burghof e Schilling (2003), o motivo para entrada no mercado está ligado à evolução da companhia: acreditam que o ápice do ciclo de vida de uma organização é listar-se em bolsa de valores. Outra vantagem, de acordo com Militão (2012), é a diversidade de financiamento e visibilidade no mercado.

Por que as empresas permanecem listadas? Há estudos que procuram correlacionar a manutenção da empresa no mercado de capitais à práticas de Governança Corporativa, entre eles citam-se Chaplinsky e Ramchand (2012), Goergen e Hinterramskogler (2013), Goktan et al. (2006), e no Brasil, Bortolon e Silva Júnior (2015b). De acordo com Charitou, Louca e Vafeas (2007), o mecanismo de governança mais influente quanto a decisão de deslistagem é o conselho de administração, pois o mesmo se encontra no ápice do centro da decisão e, por esta razão, seria o principal item a ser analisado sobre a capacidade de a empresa sobreviver na Bolsa de Valores.

Quando sair da bolsa de valores? Para Bharath e Dittmar (2010), Chaplinsky e Ramchand (2012) Louca e Vafeas (2007), Michelsen e Klein (2011), Pour e Lasfer (2013), Eid Júnior e Horng (2005) e Bortolon e Silva Júnior (2015 a), esta decisão deve ser tomada quando aumentam os custos para manter uma empresa listada, a título de exemplo Siqueira (2010) retrata que só a obrigação de publicar as demonstrações contábeis periódicas em jornais representa um acréscimo de 30\% nos custos das organizações.

Por que as empresas saem da bolsa de valores brasileira? Responder a esse questionamento e relacionar o fechamento de capital com gerenciamento de resultados é o interesse deste artigo. Nele foi estudado a terminologia deslisting ou deslistagem que, em princípio, para Bharath e Dittmar, (2010) Pour e Lasfer (2013), Michelsen e Klein (2011), Liu, Stowe e Hung (2012), Chaplinsky e Ramchand (2012), Bortolon e Silva Júnior (2015 a e b) trata-se do fechamento do capital em bolsa de valores.

A CVM, em sua Instrução Normativa 480/09, determina que no Brasil a deslistagem ocorre de duas formas: i) de ofício, quando o emissor do título de valores mobiliários descumpra, por período superior a 12 (doze) meses, suas obrigações periódicas; e ii) cancelamento voluntário, a critério do conselho de administração, que ocorrerá somente quando a empresa não tiver valores mobiliários em circulação. Estes devem ser resgatados previamente, caso não seja possível, a empresa deve obter declarações autorizativas por parte dos acionistas, como descrevem os incisos de I a IV do artigo 47. Este trabalho estudou o cancelamento voluntário, denominado deslistagem voluntária.

Esse descadastramento ou cancelamento, como definido pela CVM, não é um acontecimento exclusivamente brasileiro. Pour e Lasfer (2013) discutiram o assunto no Reino Unido, Bharath e Dittmar, (2010) e Chaplinsky e Ramchand (2012) nos EUA e Michelsen e Klein (2011) na França. Estes estudos colocam os potenciais fatores determinantes da deslistagem. Neste trabalho o fator determinante em análise será o gerenciamento de resultados, que para Cheng, Aerts e Jorissen (2010) Souza, Costa, Almeida e Bortolon (2013) está intimamente ligado a deslistagem.

Para entrada no mercado, em uma abordagem internacional, Loureiro e Silva (2020), Aslan e Kumar (2011), Bharath e Dittmar, (2010), Kim e Weisbach (2005) e Marosi e Massoud (2007) demonstram que a motivação principal para a listagem é a necessidade que a empresa apresenta de aumentar seu capital próprio. Loureiro e Silva (2020), em um estudo sobre empresas que possuem capital aberto nos EUA e outros países, relataram que as empresas que se deslistaram voluntariamente, provavelmente entraram no mercado para reequilibrar sua alavancagem. Para eles, as empresas com fluxo de caixa livre significativo, bem como as que possuem possibilidade de baixo crescimento são as mais propensas a deslistagem, pois não conseguem se beneficiar da listagem cruzada. No Brasil Eid Júnior e Horng (2005) colocam que a decisão de listagem é o resultado da avaliação entre os custos e benefícios, e eles apontam se a decisão é mais influenciada pelas variáveis que 
representam os benefícios.

Ainda na literatura internacional, alguns autores testam hipóteses para a saída de empresas em mercados pelo mundo. Park et al (2018), que estudaram o mercado japonês, explicam que a liquidez e a visibilidade financeira dos títulos das empresas deslistadas estavam nitidamente abaixo dos valores de referência. Assim, o fraco desempenho combinado a uma redução nos benefícios de listagem pode explicar como o status público da empresa aberta pode se tornar oneroso demais, aumentando os custos e diminuindo benefícios da listagem. Na China, Hu et al (2019) colocam que as pequenas e as médias empresas são mais propensas a experimentar a subvalorização de seus ativos quando comparadas às grandes corporações, estando mais propensas ao fechamento do capital, e que o as mudanças nas regras políticas (i.e., intervenção governamental) são um dos principais fatores para o fechamento de capital na bolsa chinesa após 2015. Adicionalmente, Zhou et al (2018) também na China, colocam que a pressão para a deslistagem está relacionada intimamente com mecanismos de governança corporativa, tal como o sistema de recompensação dos executivos e fraudes corporativas.

Quanto as empresas dos EUA em outros mercados, Liu, Stowe e Hung (2012) tentam explicar a deslistagem de empresas norte-americanas no mercado de capitais japonês e encontraram que a relação entre a baixa exportação e consequente baixo volume de negócios são determinantes para deslistagens voluntárias no exterior. Sobre o mercado americano propriamente dito, Chaplinsky e Ramchand (2012), mostram que as empresas maiores, mais rentáveis e com maior necessidade de captar recursos, têm menor probabilidade de serem deslistadas. Miller e Frankenthaler (2003), relatam que a instabilidade econômica nos mercados de capitais e as rigorosas exigências impostas pela Lei Sarbanes-Oxley (SOX) de 2002, forçaram as companhias abertas a avaliar a relação entre o custo e o benefício de permanecer negociando suas ações no mercado financeiro. Leuz et al. (2008) encontraram indícios de que as empresas cancelam a oferta pública em resposta ao fraco desempenho, baixas oportunidades de crescimento, e considerável aumento dos custos de transação após a adoção da lei SOX, que aumentou os custos de Governança Corporativa.

No Brasil Bortolon e Silva Júnior (2015 b) argumentam justamente o contrário, pois explicam que quanto mais avançadas são as práticas de governança corporativa menor a probabilidade de as companhias brasileiras fecharem o capital. Para Cheng, Aerts e Jorissen (2010) quando ocorrem extensas atividades de reestruturação de ativos e consequente melhoria do desempenho, ocorrerá notória diminuição da probabilidade de deslistagem.

A informação contábil representa uma das principais fontes utilizadas por diversos usuários, dentre eles, acionistas, investidores, administradores, reguladores, fornecedores, clientes e competidores, como meio de tomada de decisão, análise e controle em um mercado econômico-financeiro cada vez mais competitivo (De Moura et. al., 2017). Devido a esta utilidade, Dyck e Zingales (2004) asseguram que a informação contábil precisa ser de qualidade, uma vez que conduz a menores custos de agência, redução da assimetria da informação e proteção aos acionistas.

A qualidade da informação contábil pode ser mensurada a partir de diversos modelos capazes de capturar diferentes propriedades, entre os quais se tem gerenciamento de resultados, conservadorismo, relevância, tempestividade da informação, persistência e oportunidade (Almeida, 2010). Em diversos países, como Estados Unidos, Canadá, Reino Unido, Austrália, Finlândia e França, pesquisas estão mostrando a relevância do tema gerenciamento de resultados, conforme descrito por Stolowy e Breton (2004). Este assunto tem se tornado relevante, devido ao impacto sobre as demonstrações financeiras das organizações e, por conseguinte, influência sobre a tomada de decisão dos seus stakeholders.

Para Dechow e Skinner (2000), a definição de gerenciamento de resultados volta-se para mudanças propositais nas demonstrações contábeis das empresas. De acordo com Healy e Wahlen (1999, p. 368), "o gerenciamento de resultados ocorre quando os administradores usam julgamento sob a informação financeira e as atividades operacionais para alterar informações financeiras ou iludir alguns investidores sobre o desempenho econômico da companhia”. Em outro sentido, Martinez e Reis (2010) resumem gerenciamento de resultados como um processo de tomada de decisão sem deixar de atender às determinações 
das normas e práticas contábeis com propósito de se alcançar os objetivos esperados.

Esta pesquisa buscará aferir a qualidade da informação contábil a partir da métrica de gerenciamento de resultados pelo modelo de Kang e Sivaramakrishnan (1995). Modelo defendido por Moura, Theiss e Cunha (2014) como sendo o que apresenta maior quantidade de variáveis e que melhor reflete a realidade brasileira. Kang e Sivaramakrishnan (1995) buscaram identificar os accruals discricionários que refletem acumulações nas contas contábeis com objetivo de manipulação de resultados, ou seja, gerenciamento de resultados.

Partindo da relação em que a presença de maior qualidade das informações contábeis está em empresas com menor gerenciamento de resultados (Barth et al, 2008; Dollabona, 2011 e De Moura et al, 2016), a perspectiva deste estudo é evidenciar, conforme Francis, LaFond, Olsson e Schipper (2005), o quão a alta qualidade das informações contábeis pode ser benéfica às empresas do mercado de capitais ao proporcionar redução do custo de capital e sendo mais atrativas aos investidores. Ademais, Watts e Zimmerman (1986) indicam que a alta qualidade das informações contábeis é apreciada pelos stakeholders, posto que diminui a assimetria de informações e proporciona maior transparência.

Diante da importância econômica das temáticas sobre gerenciamento de resultados e deslistagem, este artigo traz a seguinte questão norteadora: o fechamento de capital das companhias pode provocar algum efeito sobre a prática de gerenciamento de resultados das mesmas? O objetivo é verificar a presença de tais efeitos sobre as companhias brasileiras. A amostra principal deste estudo compõe as companhias brasileiras de capital aberto, com ações negociadas na Brasil Bolsa Balcão (B3), que fecharam o capital no período de 2014 a 2017, sendo coletados dados contábil-financeiros das instituições presentes na B3 entre os anos de 2011 e 2017. A contribuição da pesquisa reside em abordar um tema pouco tratado na literatura brasileira de finanças corporativas - gerenciamento de resultados e deslistagem. Segundo, Souza, Costa, Almeida e Bortolon (2013) tais assuntos são fortemente investigados em companhias norte-americanas e pouco explorados no Brasil.

\section{Metodologia}

No que concerne aos objetivos, realizou-se pesquisa descritiva e de natureza quantitativa. A pesquisa descritiva conforme Gil (1999) tem como finalidade principal a descrição das características de determinada população ou fenômeno, ou o estabelecimento de relação entre variáveis. A abordagem quantitativa por sua vez "[...] caracteriza-se pelo emprego da quantificação tanto nas modalidades de coleta de informações, quanto no tratamento dessas através de técnicas estatísticas desde as mais simples, às mais complexas, como coeficiente de correlação, análise de regressão etc [...]" (Richardson, 1999, p.29).

A amostra principal deste estudo gira em torno das companhias brasileiras de capital aberto, com ações negociadas na Brasil Bolsa Balcão (B3), que fecharam o capital no período de 2014 a 2017. Foram identificadas neste período 70 empresas que realizaram deslistagem. Os dados contábil-financeiros coletados se referem a 355 companhias brasileiras presentes na B3 entre os anos de 2011 e 2017. Faz-se importante a coleta de dados de anos anteriores ao fechamento de capital como também de empresas que permanecem com capital aberto para se realizar comparativo entre as instituições e identificar gerenciamento de resultados anterior a deslistagem. As informações foram extraídas do banco de dados Economática ${ }^{\circledR}$.

Desconsiderou-se da amostra as instituições financeiras, empresas que apresentaram patrimônio líquido negativo e empresas que não apresentaram informações necessárias para todas as variáveis utilizadas e em todos os anos investigados. Inicialmente, calculou-se o gerenciamento de resultados com o intuito de identificar a qualidade da informação contábil das respectivas empresas. Utilizou-se o modelo de Kang e Sivaramakrishnan (1995) através do qual se permitiu estimar os accruals discricionários que representam as acumulações nas contas contábeis passíveis de manipulação dos resultados. A métrica deste modelo segue descrita no Quadro 1. Em seguida, foram calculadas as variáveis independente e de controle a serem estudadas, conforme destacado no Quadro 2. 
Quadro 1. Quadro da métrica de gerenciamento de resultados.

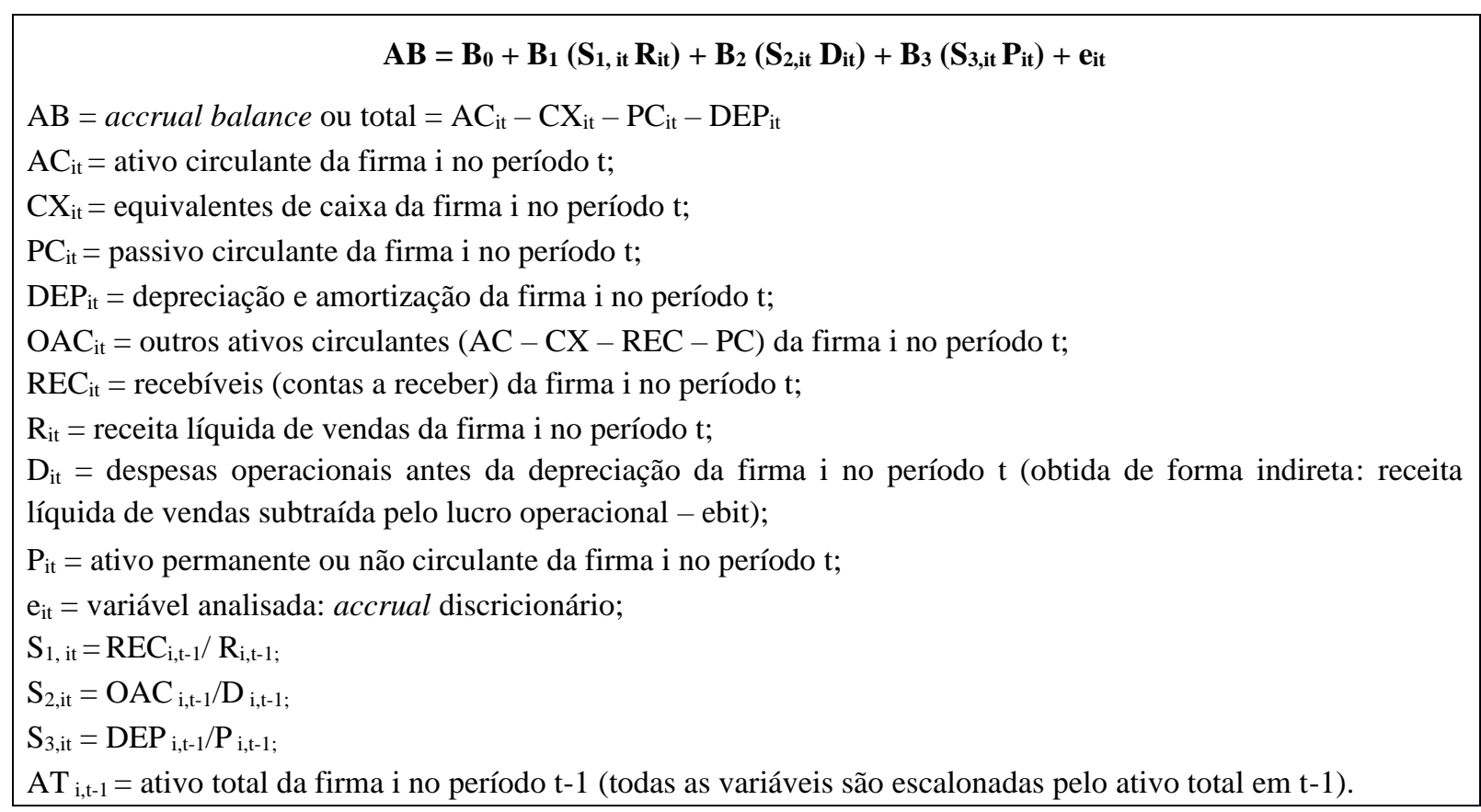

Fonte: Autores.

Quadro 2. Quadro das variáveis do estudo.

\begin{tabular}{|c|c|c|c|}
\hline Tipo & Variáveis & Métrica & Autores \\
\hline Dependente & $\begin{array}{l}\text { Gerenciamento de } \\
\text { resultados }\end{array}$ & $\begin{array}{l}\text { (GR): } \\
\text { Modelo de Kang e Sivaramakrishnan (1995) }\end{array}$ & Kang e Sivaramakrishnan (1995) \\
\hline Independente & Deslistagem & $\begin{array}{c}\text { (SIT): } \\
\text { Deslistadas }=1 \\
\text { Listadas }=0\end{array}$ & $\begin{array}{l}\text { Santos e Carvalho (2010) } \\
\text { Souza, Costa, Almeida e } \\
\text { Bortolon (2013) }\end{array}$ \\
\hline Controle & Tamanho & $\begin{array}{l}\text { (TAM): } \\
\text { Logaritmo Natural do Ativo Total }\end{array}$ & $\begin{array}{c}\text { Becker et al. (1998) } \\
\text { Watts e Zimmerman (1978) } \\
\text { Lobo e Zhou (2006) } \\
\text { Kayo et al. (2006) } \\
\end{array}$ \\
\hline Controle & Performance & $\begin{array}{c}(\mathrm{ROA}): \\
\text { Razão entre Lucro Líquido e Ativo Total }\end{array}$ & Dechow et al. (1995) \\
\hline Controle & $\begin{array}{l}\text { Concentração } \\
\text { Acionária }\end{array}$ & $\begin{array}{c}\text { (CONC): } \\
\begin{array}{c}\text { Percentual total de ações (ordinárias) em posse } \\
\text { do maior acionista. }\end{array}\end{array}$ & $\begin{array}{c}\text { Almeida (2010) } \\
\text { Herculano e Moura (2015) }\end{array}$ \\
\hline Controle & Endividamento & $\begin{array}{l}\text { (END): } \\
\text { Razão entre o passivo total e o ativo total }\end{array}$ & $\begin{array}{c}\text { Hsu (2005) } \\
\text { Morsfield e Tan (2006) }\end{array}$ \\
\hline
\end{tabular}

Fonte: Autores.

Tendo realizada a coleta dos dados, utilizou-se a análise das informações a partir da regressão linear múltipla com dados em painel não balanceados para investigar a influência das variáveis independente e de controle sobre a qualidade da informação contábil, através do software Stata®. Tal análise partiu-se do seguinte modelo econométrico:

$$
\mathrm{GR}_{i t}=\beta_{0}+\beta_{1} S I T_{i t}+\beta_{2} T A M_{i t}+\beta_{3} R O A_{i t}+\beta_{4} \mathrm{CONC}_{\mathrm{it}}+\beta_{5} E N D_{i t}+\varepsilon_{i t}
$$

Por fim, para testar a normalidade dos dados na distribuição amostral de cada variável empregada, utilizou-se o teste de Shapiro-Wilk. Além disso, para verificar a multicolinearidade entre as variáveis, foi aplicado o fator de inflação de 
variância - VIF e, a fim de verificar problemas de heterocedasticidade e autocorrelação serial, empregou-se respectivamente o teste de Wald e o teste de Wooldridge.

\section{Resultados e Discussão}

De acordo com o que foi descrito na metodologia, apresentam-se inicialmente na Tabela 1 os resultados da regressão múltipla para apuração dos accruals discricionários obtidos pelo modelo de Kang e Sivaramakrishnan (1995). A tabela mostra uma significância a $10 \%$ da variável relacionada à receita líquida $\left(\mathrm{S}_{1} \mathrm{R}\right)$ em relação aos accruals totais $(\mathrm{AB})$. Também apresenta uma significância, porém a $5 \%$, da variável referente a despesas operacionais $\left(\mathrm{S}_{2} \mathrm{D}\right)$ em relação aos accruals totais (AB). E, por fim, uma melhor significância, a 1\%, entre a variável relativa ao ativo permanente e os accruals totais (AB).

Desta regressão, calculou-se os erros padrões, identificados, portanto como os accruals discricionários, ou ainda, como GR, nomenclatura dada a variável dependente gerenciamento de resultados que será utilizada no modelo econométrico proposto. Esta variável será considerada somente em valores absolutos, conforme fundamentado por Moura, Ziliotto e Mazzioni (2016) que apontam quanto maior a distância do índice de gerenciamento de resultados de zero, maior será o ajustamento das contas contábeis, não importando se apresentarem valores positivos ou negativos.

Junto à regressão múltipla para apuração dos accruals discricionários foi aplicado o teste de Chow para verificar qual modelo seria o mais apropriado, o de efeitos fixos ou pooled. Rejeitou-se a hipótese nula do modelo pooled, indicando que o modelo de efeitos fixos é o mais apropriado à regressão. Ainda foi aplicado o teste de Hausman, o qual rejeitou a hipótese nula de efeitos aleatórios, indicando novamente o modelo de efeitos fixos como o mais apropriado. O teste de Wald para dados em painel identificou problema de heterocedasticidade e o teste de Wooldridge identificou problema de autocorrelação. Tais problemas foram tratados pela adoção dos estimadores robustos de White.

Tabela 1. Regressão modelo KS (1995) de apuração dos accruals discricionários

\begin{tabular}{|c|c|c|c|c|c|c|}
\hline $\mathbf{A B}$ & Coeficiente & $\begin{array}{c}\text { Erros Padrões } \\
\text { Robustos } \\
\end{array}$ & $\mathbf{t}$ & $\mathbf{P}>\mathbf{t}$ & \multicolumn{2}{|c|}{ [95\% Intervalo de Confiança $]$} \\
\hline$S_{1} R$ & $0.081 *$ & 0.04 & 1.69 & 0.091 & -0.01 & 0.17 \\
\hline $\mathrm{S}_{2} \mathrm{D}$ & $0.033 * *$ & 0.01 & 2.05 & 0.041 & 0.00 & 0.06 \\
\hline $\mathrm{S}_{3} \mathrm{P}$ & $-0.80^{* * *}$ & 0.22 & -3.55 & 0.000 & -1.25 & -0.35 \\
\hline Constante & $0.04 * * *$ & 0.01 & 4.18 & 0.000 & 0.02 & 0.06 \\
\hline $\begin{array}{l}\text { Número } \\
\text { Níveis d } \\
\text { AB: } a c c \text { 1 } \\
\mathrm{S}_{1} \mathrm{R}: \text { rece } \\
\mathrm{S}_{2} \text { D: des } \\
\mathrm{S}_{3} \mathrm{P} \text { : ativ } \\
\end{array}$ & $\begin{array}{l}\text { bservações: } 1 \\
\text { ificância: } 1 \% \\
\text { totais } \\
\text { quida } \\
\text { operacionais } \\
\text { manente }\end{array}$ & $* *), 5 \%(* *), 10 \%$ & & & & \\
\hline
\end{tabular}

Fonte: Dados da Pesquisa.

Após o cálculo da variável dependente GR, foi realizado o teste para verificar a diferença das variáveis do modelo econométrico proposto entre os grupos de empresa deslistada e empresa listada. A princípio aplicou-se o teste de normalidade de Shapiro-Wilk (SW) a fim de verificar se essas variáveis seguem uma distribuição normal. A Tabela 2 apresenta os resultados do teste de normalidade. O teste demonstra que todas as variáveis não apresentam uma distribuição normal, pois todos os resultados foram significativos, rejeitando-se a hipótese nula de normalidade na distribuição. Como a distribuição não é normal, utilizou-se o teste não paramétrico de Mann-Whitney para verificar as diferenças das variáveis entre os dois grupos. 
Tabela 2. Teste de normalidade de Shapiro-Wilk.

\begin{tabular}{lccccc}
\hline Variável & $\mathbf{N}$ & $\mathbf{W}$ & $\mathbf{V}$ & $\mathbf{z}$ & Prob>z \\
\hline GR & 1879 & 0.71 & 318.36 & 14.62 & 0.00 \\
SIT & 2485 & 0.99 & 3.89 & 3.48 & 0.00 \\
TAM & 2058 & 0.91 & 107.75 & 11.91 & 0.00 \\
ROA & 2058 & 0.01 & 1205.39 & 18.06 & 0.00 \\
CONC & 2056 & 0.93 & 77.80 & 11.08 & 0.00 \\
END & 2058 & 0.97 & 26.54 & 8.34 & 0.00 \\
\hline
\end{tabular}

Fonte: Dados da Pesquisa.

A Tabela 3 evidencia os resultados do teste não paramétrico de Mann-Whitney. Diante dos resultados, todas as variáveis mostraram significância no teste em questão, rejeitando a hipótese nula de igualdade de mediana entre as empresas deslistadas e empresas listadas. Portanto, com relação a todas as variáveis propostas é possível se inferir sobre as diferenças apresentadas na Tabela 4 entre os dois grupos.

Tabela 3. Teste de Wilcoxon-Mann-Whitney.

\begin{tabular}{|c|c|c|c|c|}
\hline Variável & Tipo de Empresa & $\mathbf{N}$ & $\mathbf{Z}$ & Prob > $|z|$ \\
\hline GR & $\begin{array}{l}\text { Deslistada } \\
\text { Listada }\end{array}$ & $\begin{array}{c}185 \\
1694\end{array}$ & -2.53 & $0.01 * * *$ \\
\hline TAM & $\begin{array}{l}\text { Deslistada } \\
\text { Listada }\end{array}$ & $\begin{array}{c}186 \\
1872\end{array}$ & 6.16 & $0.00 * * *$ \\
\hline $\mathrm{ROA}$ & $\begin{array}{l}\text { Deslistada } \\
\text { Listada }\end{array}$ & $\begin{array}{c}186 \\
1872\end{array}$ & 2.45 & $0.01 * * *$ \\
\hline $\mathrm{CONC}$ & $\begin{array}{l}\text { Deslistada } \\
\text { Listada }\end{array}$ & $\begin{array}{c}240 \\
1816\end{array}$ & -12.85 & $0.00 * * *$ \\
\hline END & $\begin{array}{l}\text { Deslistada } \\
\text { Listada }\end{array}$ & $\begin{array}{c}186 \\
1872\end{array}$ & 5.81 & $0.00 * * *$ \\
\hline
\end{tabular}

Níveis de significância: $1 \%(* * *), 5 \%(* *), 10 \%(*)$. Fonte: Dados da Pesquisa.

A Tabela 4 apresenta a estatística descritiva das variáveis propostas no modelo econométrico a partir da identificação da empresa por sua listagem em bolsa de valores (deslistada ou listada). Os resultados da estatística descritiva demonstram que as empresas deslistadas em média gerenciam (GR) mais seus resultados (0.037) em comparação às empresas listadas (0.035).

Com relação às demais variáveis, as empresas deslistadas apresentaram, em média, tamanho (TAM) menor (13.538) quando comparados às empresas listadas que tiveram, em média, tamanho maior (14.644). Análogo aos valores de tamanho, a rentabilidade (ROA) se mostrou, em valores médios, menor para empresas deslistadas (-3.608) e maior para empresas listadas (-0.007). Assim como o endividamento (END) que também apresentou valor médio menor para empresas deslistadas (0.179) e valor médio maior para empresas listadas (0.261). Quanto à concentração acionária (CONC), as empresas deslistadas apresentaram valor médio significativamente superior (70.730) quando comparado ao valor médio das empresas listadas (44.757). 
Tabela 4. Estatística descritiva por classificação da empresa quanto à listagem em bolsa de valores.

\begin{tabular}{lcccccccccc}
\hline \multicolumn{9}{c}{ Deslistada } \\
\hline Variável & $\mathbf{N}$ & Média & $\begin{array}{c}\text { Desvio } \\
\text { Padrão }\end{array}$ & Min & Max & N & Média & $\begin{array}{c}\text { Desvio } \\
\text { Padrão }\end{array}$ & Min & Max \\
\hline GR & 185 & 0.037 & 0.021 & 0.000 & 0.171 & 1694 & 0.035 & 0.026 & 0.000 & 0.482 \\
TAM & 186 & 13.538 & 2.532 & 4.949 & 18.595 & 1872 & 14.644 & 2.409 & 2.724 & 20.740 \\
ROA & 186 & -3.608 & 49.141 & -670.188 & 0.727 & 1872 & -0.007 & 0.710 & -20.160 & 0.77 \\
CONC & 240 & 70.730 & 25.936 & 9.998 & 100 & 1816 & 44.757 & 26.466 & 0.138 & 100 \\
END & 186 & 0.179 & 0.171 & 0.000 & 0.573 & 1872 & 0.261 & 0.187 & 0.000 & 0.870 \\
\hline
\end{tabular}

Fonte: Dados da Pesquisa.

A seguir, a Tabela 5 apresenta a matriz de correlação de Pearson das variáveis propostas no estudo com o objetivo de avaliar a correlação entre elas. Ao nível de $10 \%$ de significância, observa-se uma correlação significativa e negativa de -0.20 entre gerenciamento de resultados (GR) e tamanho da empresa (TAM). Assim, entre as empresas da amostra, os resultados sinalizam que as maiores empresas, apresentaram menores índices de gerenciamento de resultados. Outra correlação significativa, e também negativa, é entre gerenciamento de resultados (GR) e endividamento (END) ao valor de -0.24 . Tais constatações confirmam o que foi descrito no estudo de Almeida (2010), no qual identificou correlação significativa e negativa entre as variáveis gerenciamento de resultados e tamanho e entre gerenciamento de resultados e endividamento.

Corroborando com a matriz de correlação apresentada, observa-se na Tabela 6 o resultado da regressão do modelo econométrico estimado. Destaca-se os coeficientes da regressão, capazes de possibilitar a análise do poder das variáveis independentes e de controle sobre o gerenciamento de resultados (variável dependente). Pode-se observar ainda na Tabela 6 que $\mathrm{o} \mathrm{R}^{2}$ foi de apenas 7,6\%. Porém, o valor encontrado está correlato à pesquisa de Almeida (2010) que identificou o $\mathrm{R}^{2}$ entre $7,36 \%$ e $22,28 \%$.

Tabela 5. Correlação entre variáveis.

\begin{tabular}{lllllll}
\hline & GR & SIT & TAM & ROA & CONC & END \\
\hline GR & 1.00 & & & & & \\
SIT & 0.02 & 1.00 & & & & \\
TAM & $-0.20^{*}$ & $-0.13^{*}$ & 1.00 & & & \\
ROA & $-0.04^{*}$ & $-0.06^{*}$ & $0.10^{*}$ & 1.00 & & \\
CONC & -0.01 & $0.30^{*}$ & $-0.27^{*}$ & $-0.05^{*}$ & 1.00 & \\
END & $-0.24^{*}$ & $-0.12^{*}$ & $0.46^{*}$ & 0.03 & $-0.08^{*}$ & 1.00 \\
\hline
\end{tabular}

Nível de significância 10\% (*). Fonte: Dados da Pesquisa.

Sobre os testes aplicados anteriores a regressão, foi realizado o teste VIF, através do qual não constatou problemas de multicolinearidade entre as variáveis independentes, sendo todos os valores VIF abaixo de 10. Ainda se realizou os testes de Wald e de Wooldridge para dados em painel, identificando somente problema de heterocedasticidade. Tal problema foi tratado com a utilização dos estimadores robustos de White. Aplicou-se os testes de Breush Pagan, rejeitando-se a hipótese nula de efeitos pooled, indicando modelo de efeitos aleatórios.

Em relação aos coeficientes da equação apresentados na Tabela 6, a relação principal do nosso modelo econométrico entre a variável dependente gerenciamento de resultados (GR) e a variável independente dummy de deslistagem (SIT) não apresentou coeficiente estatisticamente significativo. Devido a isso, não se pode afirmar que a deslistagem de determinada empresa da amostra reflete sobre seu gerenciamento de resultados. O resultado difere do encontrado por Santos e Carvalho 
(2010) e Souza, Costa, Almeida e Bortolon (2013) ao identificarem que o fechamento de capital influencia o gerenciamento de resultado para cima, devido ao sinal positivo encontrado.

Tabela 6. Resultado da regressão do modelo econométrico.

\begin{tabular}{ccc}
\hline Variáveis & Coeficiente & Estatística-z \\
\hline Constante & $0.0705^{* * *}$ & 6.36 \\
SIT & 0.0005 & 0.14 \\
TAM & $-0.0018^{* * *}$ & -2.46 \\
ROA & 0.0080 & 0.85 \\
CONC & -0.00002 & -0.91 \\
END & $-0.0226 * * *$ & -4.51 \\
\hline N (Observações) & 1795 & \\
N (Grupos) & 308 & \\
R $^{2}$ & 0.076 & \\
VIF & $<10$ & \\
Teste Wooldridge (Prob>F) & 0,631 & \\
Teste Wald (Prob>chi2) & 0,000 & \\
Teste de Breush Pagan & 0,000 & \\
(Prob>chi2) &
\end{tabular}

Níveis de significância: $1 \%(* * *), 5 \%(* *), 10 \%(*)$. Fonte: Dados da Pesquisa.

Observando as variáveis de controle propostas no modelo, identifica-se uma relação significativa e negativa de 0.0018 entre gerenciamento de resultados (GR) e tamanho da empresa (TAM). O resultado é similar e alinha-se ao estudo de Santos e Carvalho (2010), no qual revelam que empresas maiores possuem maior monitoramento de suas atividades por parte de acionistas e analistas e, consequentemente, apresentam menor gerenciamento de resultados.

No que diz respeito ao grau de endividamento da empresa (END), obteve-se uma relação significativa e negativa de 0.0226 em referência ao gerenciamento de resultados (GR). Tal resultado também corrobora com o estudo de Souza, Costa, Almeida e Bortolon (2013) ao indicarem que a presença de maior número de credores devido a um maior grau de endividamento acarreta gerenciamento de resultados para baixo.

No que tange a relação entre performance (ROA) e gerenciamento de resultados (GR), não foi obtida significância para esta relação. Com tudo, este estudo alinha-se a Dechow, Sloan e Sweeney (1995) quando os autores afirmam haver correlação entre accruals discricionário da empresa e sua performance, apresentando ambiguidade para o sinal esperado. Na Tabela 5 de correlação entre as variáveis, pode-se confirmar o alinhamento dos estudos. Relativo à concentração acionária, o resultado alinha-se ao estudo de Moura, Macêdo, Mazzioni e Kruger (2016) que ao analisarem a relação entre concentração acionária e gerenciamento de resultados no mercado brasileiro no período de 2008 a 2014, não identificaram significância entre as variáveis.

\section{Conclusão}

Para se chegar aos resultados encontrados, optou-se por uma pesquisa descritiva de natureza quantitativa com fonte de dados da Economática ${ }^{\circledR}$, tendo como amostra principal as empresas que fecharam o capital no período de 2014 a 2017. Este período exemplifica o recente declínio em quantidade de empresas no mercado de capitais brasileiro, com os crescentes e recentes descadastramentos, compreendendo tanto períodos de estabilidade econômica quanto períodos de crise na financeira.

Elegeu-se o modelo desenvolvido por Kang e Sivaramakrishnan (1995), conhecido por KS, para se calcular o gerenciamento de resultados e consequentemente para se medir a qualidade da informação contábil das respectivas empresas. A variável gerenciamento de resultados ou accruals discricionários, calculada a fim de compor o modelo econométrico proposto, é considerada somente em seus valores absolutos, sendo possível identificar que quanto maior a distância do accruals 
encontrado de zero, maior será o ajustamento das contas contábeis.

Esta pesquisa buscou explicar, por meio da regressão linear múltipla com dados em painel, a relação existente entre gerenciamento de resultados (GR) e deslistagem (SIT) em conjunto com as variáveis de controle tamanho, performance, concentração acionária e endividamento. Foi encontrada evidência sobre tamanho das empresas que indica possuir relação significativa e negativa com o gerenciamento de resultados, onde as maiores empresas da amostra apresentaram menores índices de gerenciamento de resultados e, por conseguinte, maior qualidade da informação contábil. Esta evidência corrobora com o estudo de Santos e Carvalho (2010).

Outra evidência encontrada foi entre a relação de endividamento e gerenciamento de resultados. O resultado aponta para uma relação também significativa e negativa, na qual empresas mais endividadas gerenciam menos seus resultados, alinhando-se a pesquisa de Souza, Costa, Almeida e Bortolon (2013). De modo contrário, não se pode confirmar a relação entre gerenciamento de resultados e deslistagem por não ter sido possível identificar significância nesta relação.

Como sugestões para pesquisas futuras podem ser apontadas a adoção de um período maior de estudo das variáveis sugeridas, a utilização de outros modelos de mensuração da proxy de gerenciamento de resultados, e, por fim, direcionar estudos para outras formas de descadastramento, incluindo as involuntárias, quando as empresas contrariam exigências da CVM.

\section{Referências}

Achleitner, A-K., et al. (2013). Private equity acquisitions of continental European firms: the impact of ownership and control on the likelihood of being taken private. European Financial Management, 19(1), 72-107.

Almeida, J. E. F. D. (2010). Qualidade da informação contábil em ambientes competitivos. Doctoral dissertation, Universidade de São Paulo.

Antunes, Gustavo Amorim et al. (2008). Empresas estatais federais e empresas do novo mercado da Bovespa: um estudo comparativo acerca da qualidade da informação contábil utilizando dados em painel. In: Congresso USP de Controladoria e Contabilidade,

Aslan, H., \& Kumar, P. (2011). Lemons or cherries? Growth opportunities and market temptations in going public and private. Journal of Financial and Quantitative Analysis, 46(2), 489-526.

Barth, M. E. et al. (2006). Accounting quality: International accounting standards and US GAAP. Manuscript, Stanford University.

Barth, M. E., Landsman, W. R., \& Lang, M. H. (2008). International accounting standards and accounting quality. Journal of accounting research, 46(3), 467498.

Bharath, S. T. \& Dittmar, A. K. (2010) Why do firms use private equity to opt out of public markets?. The Review of Financial Studies, 23(5), 1771 -1818.

Bortolon, P. M., \& Da Silva Junior, A. (2015a). Fatores determinantes para o fechamento do capital de companhias listadas na BM\&FBOVESPA. Revista Contabilidade \& Finanças, 26(68), 140-153.

Bortolon, P. M., \& Da Silva Junior, A. (2015b). Deslistagem de companhias brasileiras listadas na bolsa de valores: evidências empíricas sobre a Governança Corporativa. Brazilian Business Review, Edição Especial, p. 97-124.

Bortolon, P. (2013). Porque as empresas brasileiras adotam estruturas piramidais de controle. Revista Base (Administração e Contabilidade) da UNISINOS, 10(1).

Burghof, H.-P., \& Schilling, D. (2003). Going private as corporate governance transaction. Betriebswirtschaftliche Forschung und Praxis, 55(2), 117-136.

Chaplinsky, S., \& Ramchand, L. (2012). What drives delistings of foreign firms from US Exchanges? Journal of International Financial Markets, Institutions and Money, 22(5), 1126-1148.

Charitou, An., Louca, C., \& Vafeas, N. (2007). Boards, ownership structure, and involuntary delisting from the New York Stock Exchange. Journal of Accounting and Public Policy, 26(2), 249-262.

Cheng, P., Aerts, W., \& Jorissen, A. (2010). Gerenciamento de lucros, reestruturação de ativos e a ameaça de fechamento de bolsa em um regime regulatório baseado em lucros. Governança Corporativa: Uma Revisão Internacional, 18(5), 438-456.

Dallbona, L. F. (2011). Qualidade das informações contábeis de empresas negociadas na BM\&FBovespa: aderência às normas internacionais de contabilidade. Blumenau. Programa de Pós-Graduação em Ciências Contábeis, Universidade Regional de Blumenau (Dissertação de Mestrado).

De Moura, et al. (2017). Determinantes da qualidade da informação contábil em grandes companhias abertas listadas na BM\&FBOVESPA. Revista de Educação e Pesquisa em Contabilidade (repec), 11(3). 
De Moura, G. D., et al. (2016). Análise da relação entre gerenciamento de resultados e custo de capital em empresas brasileiras listadas na Bm\&FBovespa. Revista Catarinense da Ciência Contábil, 15(44), 9-23.

De Souza, J. A. S., et al. (2013). Determinantes e consequências do fechamento de capital nas práticas de gerenciamento de resultados. Revista Evidenciação Contábil \& Finanças, 1(1), 38-57.

Dechow, P. M. \& Skinner, D. J. (2000) Earnings management: Reconciling the views of accounting academics, practitioners, and regulators. Accounting horizons, 14(2), 235-250.

Dechow, P. M., Sloan, R. G., \& Sweeney, A. P. (1995). Detecting earnings management. Accounting review, 70(2), $193-225$.

Dias de Moura, G., Theiss, V. \& Da Cunha, P. R. (2014). Ativos intangíveis e gerenciamento de resultados: uma análise em empresas brasileiras listadas na BM\&FBovespa. Revista Base (Administração e Contabilidade) da UNISINOS, 11(2).

Dyck, A., \& Zingales, L. (2004). Private benefits of control: An international comparison. The journal of finance, 59(2), 537-600.

Eid Júnior, W., \& Horng, W. J. (2005). A saída: uma análise da deslistagem na Bovespa. Working paper. Fundação Getúlio Vargas.

Francis, J., LaFond, R., Olsson, P., e Schipper, K. (2005). The market pricing of accruals quality. Journal of accounting and economics, $39(2), 295-327$.

Gil, Antônio Carlos. (1999). Pesquisa social: métodos e técnicas. Atlas.

Goktan, M. S., Kieschnick, R., \& Moussawi, R. (2006). Corporate governance and corporate survival. University of Texas at Dallas, United States of America.

Healy, Paul M \& Wahlen, James M. (1999). Uma revisão da literatura de gerenciamento de resultados e suas implicações para a definição de padrões. Horizontes contábeis, 13(4), 365-383.

Hu, G., Lin, J. C., \& Owen Wong, M. Y. (2019). Why have many U.S.-listed Chinese firms announced delisting recently? Global Finance Journal, 41, 13-31.

Kang, Sok-Hyon \& Sivaramakrshnan, K. (1995). Issues in testing earnings management and an instrumental variable approach. Journal of accounting Research, 33(2), 353-367.

Kim, W., \& Weisbach, M. (2005). Do firms go public to raise capital? Massachusetts, National Bureau of Economic Research.

Li, J., \& Zhou, J. (2006). Earnings management and delisting risk of initial public offerings. Simon School, University of Rochester, Research Paper Series.

Loureiro, G. \& Silva, S. (2020). The impact of cross-delisting from the U.S. On firm' financial constraints. Journal of Business Research, 108, $132-146$.

Malacrida, M. J. C., \& Yamamoto, M. M. (2006). Governança corporativa: nível de evidenciação das informações e sua relação com a volatilidade das ações do Ibovespa. Revista Contabilidade \& Finanças, 17, 65-79.

Marosi, A., \& Massoud, N. (2007). Why do firms go dark?. Journal of Financial and Quantitative Analysis, 42(2), $421-442$.

Martinez, I., \& Serve, S. (2011). The delisting decision: The case of buyout offer with squeeze-out (BOSO). International Review of Law and Economics, 31(4), 228-239.

Michelsen, M., \& Klein, C. (2011). "Privacy please!” The public to private decision in Germany. Review of Managerial Science, 5(1), 49-85.

Militão, F. (2012). Fechamento voluntário de capital e o investidor minoritário na opinião dos profissionais de mercado. Tese de Doutorado. Dissertação de Mestrado Profissional, Fundação Pedro Leopoldo, Minas Gerais, Brasil.

Monezi, M. (2004). Como estimular a abertura de capital. Jus Navigandi, 8(397).

Nóbrega, M. et al. (2000). O mercado de capitais: sua importância para o desenvolvimento e os entraves com que se defronta no Brasil. Publicações Bovespa. São Paulo.

Pagano, M., Panetta, F., \& Zingales, L. (1998). Why do companies go public? An empirical analysis. The journal of finance, 53(1), 27-64.

Park, J., Shiroshita, K., Sun, N., \& Park, Y. W. (2018). Involuntary delisting in the Japanese stock market. Managerial Finance, 44(9), $1155-1171$.

Paulo, E., \& Martins, E. (2007). Análise da qualidade das informações contábeis nas companhias abertas. Anais do Encontro da Associação Nacional de PósGraduação e Pesquisa em Administração, São Paulo, SP, Brasil, 31, 1-16.

Pour, E. K., \& Lasfer, M. (2013). Why do companies delist voluntarily from the stock market? Journal of Banking \& Finance, 37(12), 4850-4860.

Richardson, R. J. et al. (1999). Pesquisa social: métodos e técnicas. (3a ed.), Atlas.

Santos, I. T., \& De Carvalho, A. G. (2010). Gerenciamento de resultados em fechamento de capital. In: $32^{\circ}$ Meeting of the Brazilian Econometric Society.

Stolowy, H., \& Breton, G. (2004). Accounts manipulation: A literature review and proposed conceptual framework. Review of accounting and finance, 3(1), 592.

Watts, R. L., \& Zimmerman, J. L. (1986). Positive accounting theory. Prentice-Hall Inc., https://ssrn.com/abstract=928677.

Zhou, F., Zhang, Z., Yang, J., Su, Y., \& An, Y. (2018). Delisting pressure, executive compensation, and corporate fraud: Evidence from China. Pacific-Basin Finance Journal, 48, 17-34. 